\title{
LA VOIE VEINEUSE: SOUS-CLAVIERE EN ANESTHESIE
}

\section{KeÉri-SzÁntó, M D , Gư Fortin, M.D., et André RrouX, M.D *}

Cette nouvelde vore d'accès à la circulation veineuse a été préconisée par Aubainac (1). L'étude puatıquée sur cadavres par l'un de nous (2) a confirmé c u'il s'agıt d'une technıque fiable et précıse. Afin d'évaluer l'application courante ce cette méthode, à la salle d'opératıon, au dispensaire d'urgence, ou dans les locaux préposés aux soins immédıats des opérés, nous avons poussé cette enquête dans le but d'analyser les poirts suivants: (1) Douleur provoquée par la cannulation, (2) L'attitude du personnel médical en général envers cette procédure; (3) La proportion des échecs, (4) La proportion et le danger des erreurs de technıques. Uné étude des cent premiers cas nous permet de préciser d'avantage les points énumérés ci-dessus

\section{Matériel et MÉThOdes UTILISÉS}

Cette étude a été réalisée à la salle d'opération et au dispensaure d'urgence de notre hôpital, sans sélection de patients. Nous nous sommes appliqués à utılıser de routine la vore vemeuse sous-clavière, avant ou 'après le début de l'anesthésie La cannulation a été pratıquée d'un seul côté. En' cas d'échec, nous nous sommes tenus à une seule manœuvre de reprise. Au début les patients étaient transportés sur civière à la salle de recouvrance avec l'aiguille in situ pour la thérapıe parentérale. L'arguılle étast toujours enleyée avant le retour du malade à sa chambre Chez l'adulte, nous avons utlisé un trocard de trois pouces de longueur de calıbre 18 lorsqu'une transfusion 'étalt anticipée et de calıbre 22 pour l'administration intraveineuse des anesthésıques. Chez les nouveaux-nés et les enfants en bas âge, des aiguilles proportionnellement plus courtes et de calıbre 21 ont donné un rendement satisfaisant.

Après luı avoir fait subır de multiples modificatıons légères, nous présentons la technique des injections sous-clavières telle que nous la pratiquons à l'heure actuelle

Deux centımètres cubes de novocaine à une concentratıon de 2 pour cent sont aspirés dans une seringue, montée d'une aigulle de calıbre 24 . Un bouton intradermıque est soulevé au nıveau du bord inférıeur'de la clavioule, à l'union de ses tiers moyen et interne. Les tissus sous-jacents sont infiltrés en profondeur. La même seringue est maintenant montée d'une aigulle de troıs pouces de longueur et de calıbre 18 L'aigulle est introduite au niveau du bouton intra-dermique en lu donnant une inclinaison vers l'extrémité céphalique et est dırigée vers le plan dorsal et médian tout en longeant le bord inférieur de la clavicule. L'aiguille, rentrée de la façon décrite, traverse lle ligament costo-claviculaire en donnant la sensation de la ponction dureménenne. L'opérateur, à ce moment, doit faire avancer l'aiguille lentement en pratıquant une légère aspiration sur la serıngue. $\mathrm{A}$ quelques millimètres au-delà du lıgament costo-claviculaire, la veine sousclavière est pénétrée et le sang reflue dans la seringue. Une aspiration continue,

"Service d'Anesthésie, Hôpital Notre-Dame, Montréal, PQ. 
en faisant farre à l'arguille un tour complet sur elle-même, confirme la pénétratıon complète du biseau de l'arguille à l'intérieur de la veıne. Cette dernère manœuvre est essentielle afin d'éviter l'extravasation du liquide de perfusion. Afin d'éviter l'embolıe gaseuse, complıcation que nous croyons fort peu probable mais que nous devons tout de même envisager par mesure de précaution, l'échange de la seringue pour la tubulure du flacon d'infusion doit se faire rapidement pendant l'expiration. L'échange peut se faire aussı en demandant au patient de retenir sa respuration. L'argulle est im nobilısée par un diachilon fixé en sangle autour de sa tige et crossé au devant. Cette façon de fixer l'angulle l'empêche de progresser en profondeur au cas où elle serat heurtée accidentellement. L'arguille se trouve maintenant fixée fermement à son extrémité distale par le ligament costo-clavicularre qui est relativement beaucoup plus dense que les tassus sous-jacents. Autour de ce point d'appui, tandis que la partie visible de l'aiguille est très mobile, sa portion intra-vasculaure oscille dans un petıt rayon sans subır de déplacement important La tubulure d'infusion est fixée à l'épaule avec un diachılon de façon à éviter une tractıon sur l'arguılle. A part c'élımination de linfiltration locale, 1 n'y a pas de variation de technique lorsqu'on utilise une argulle de calıbre 22. Pendant la perfusion la positıon de l'arguille doıt être vérifiée pérıodıquement par aspiration avec une seringue ou par sıphonage du sang vemeux en abaissant le flacon perfuseur à un nıveau plus bas que celuı de l'aiguille Cette même précaution doit être observée avant chaque administration de médıcaments à la serıngue L'épreuve est répétée après chaque changement de position du patient.

L'évaluation de la méthode s'est poursurvie dans la phase post-opératorre par la recherche du nombre et de la gravité des complications locales et générales En outre, on interrogea la mémoure du patient sur les sensations éprouvées au moment de la ponction et après l'intervention. Toute complication, même douteuse, fut l'objet d'une investgation marquée d'un examen physique complet et d'études radıologiques, au besoin en séries, de la cage thoracique.

\section{RÉSULtats}

Le tableau I indıque le nombre de patients, les lımites d'âges, le nombre de tentatives et la fréquence des échecs Le tableau II indique la fréquence et la nature des complıcations rencontrées dans cette série.

Bien que les exigeances d'investigation au cours de cette présente série furent beaucoup plus rigides que celles imposés au cours du travall précité, le pourcentage des cannulations réussies est le même. Ce résultat est probablement attribuable à part égale à l'expérience acquise et à l'impression que la cannulation de la veine chez le vivant est beaucoup plus facle que chez le cadavre $\mathrm{Au}$ point de vue pratique, lorsque les restrictions imposées par les exigeances de l'investigation clinique sont relevées, nous sommes assurés d'une réussite dans presque tous les cas d'urgence nécessitant une cannulation de la veıne sousclavière. Puısqu'il s'agit principalement de comparer l'injection sous-clavière aux autres méthodes intraveıneuses usuelles, nos complicatıons ont été déterminées par des critères très rigides. Au cours de cette étude la seule vraie complication chez une patiente fut la persistance d'une douleur cutanée assez vive à 
TABLEAU I

\begin{tabular}{lr}
\hline Ages & Nombres dé cas \\
\hline Mons de 2 ans & 4 \\
Moins de 10 an:, & 4 \\
10 ans et plus & 105 \\
Nombres de cas réussis en un seul essal & $88-78 \%$ \\
Nombres de cas réussis au deuxième essar & $18-16 \%$ \\
Echecs & $7-6 \%$ \\
\hline
\end{tabular}

TABLEAU II

\begin{tabular}{lr}
\hline Complications & Nombres de cas \\
\hline Souvenur de douleur associé à la procédure & 11 \\
Douleur persistante & 4 \\
Pénétration accidentelle de l'artère sous-clavière & 3 \\
Aiguille délogét & 10 \\
Ponction pleurale accidentelle & 2
\end{tabular}

l'endrott de la ponction Cette douleur persista pendant quatre jours. Dans ce cas, un examen physıque détallé n’a révélé aucun sıgne objectıf ce qui nous fait croire à une lésion discrète d'un nerf sensitıf cutané ou encore à une manifestation psychosomatique Un autre patient éprouva une douleur semblable au moment de I'introduction de l'aigulle. Elle disparut instantanément après le désinsertion de l'arguille. Là où l'épreuve der siphonage fut négatıve, on présuma que l'arguille ètait délogée Cette manœuvre de siphonage répétée pérodıquement a souvent contribué à éviter l'apparition d'une tuméfaction. Dans les quelques cas où une tuméfaction apparut, la collection de Iıquide fut toujours en avant de l'aponévrose cervicale superficielle et ne provoque pas d'obstruction respiratore nı séquelles ultérieures, Cette complication fut chaque fors associée à un changement de position. L'absorption des lıquides extravasés accidentellement dans la région du triangle sous-clavicularre est très rapide. En effet l'absorption des agents anesthésıques dans une telle circonstance a été tellement rapıde que la narcose s'est réalisée sans la nécessité d'augmenter le dosage d'une façon appréciable La pénétration de l'arguille dans l'artère sous-clavière a été décelée instantanément par la couleur et la pression pulsatile du sang. La perforation de l'artère ne fut survie d'aucune complicatıon après l'extraction de l'aiguille. La plèvre fut perforée au cours d'une tentative d'mjection sous-clavière chez un patient déjà en décubitus latéral. Dans un autre cas, la perforation pleurale est survenue pendant une installation en position latérale après.cannulation mitiale en décubitus dorsal. Ċes deux cas ne furent suvis d'aucune complication après l'enlèvement de l'aiguille.

Quelques-uns des avantages et désavantages de la technique ne peuvent être présentés sous forme de lableau. synoptıque Quelques patients ont refusés cette cannulation, En général, la méthode a été bien accuellie par le personnel médıcal de notre hôpital

Un des avantages les plus utiles de la technique découle du fait qu'au besoin l'anesthésiste peut réaliser une injection intraveineuse sans délai indépendam- 
ment de l'état général et de l'âge du patient. Ceci permet de déférer les injections intravemeuses habituellement installées à titre prophylactiques. On' élimine beaucoup de délaı dans la préparation des patients à la phase d'induction de l'anesthésie, ce qui est d'une grande valeur chez les patients qui présentent de l'appréhension. La technique exclut les inconvéments d'une intravemeuse enfoure sous les draps, d'un support toujours nussible pour le chirurgien et dangereux pour le bras du patient et les dangers et ennus d'une installation intraveineuse au pied du patient. La région sous-clavière est habituellement accessible à l'anesthésıste dans presque toutes les intervention:s et l'aigulle peut être protégée adéquatement même au cours d'une laparatomie sus-ombilicale. La vasoconstriction veineuse périphérique survient souvent'à un moment précaire et entrave le bon fonctionnement d'une intraveineuse installée au niveau d'une extrémité. Puisque la veine sous-clavière ne participe pas à cette vasoconstrictoon, c'est là précısément l'avantage princıpal de la voıe veıneuse sous-clavière par opposition à la vore veineuse périphérique. En effet, ce phéncmène a fait l'objet d'une communication récente (3) en rapport avec la cannt lation de la veine brachıle céphalıque Bien que cette veune présente l'avantage de ne pas participer à un collapsus périphérique généralısé, sa cannulation exige cependant une dissection.

La cannulation de la veine sous-clavière est une technique de grande valeur à tout point de vue et s'acquiert facilement Elle présente de multiples avantages à la salle d'opération et élımine une source de cléla dans la préparation des patients à l'intervention. La maîtrise de cette technıque augmentera l'assurance de l'anesthésiste et bénéficiera aux enfants et aux adultes appréhensifs Elle contribuera à éviter des délaıs importants et à dimınuer ainsı le taux de mortalıté dans les cas d'urgence qui exigent une thérapie parentérale ımmédiate

\section{SOMMAIRE}

La cannulation de la veine sous-clavière fut réalisée avec succès dans une proportion de plus de 90 pour cent sur une sérne de patıents pusés au hasard à la salle d'opération. La proportion et la nature des difficultés rencontrées au cours de cette procédure sont présentées et discutées Une maîtrise de cette technıque très utile contribuera tout probablement à sauver la vie de nombreux patients dans les cas d'urgence

\section{ADDENDUM}

Depurs la présentation de ce travail pour publication, le nombre de cas se chiffre maintenant à trois cent. Malheureusement parmı ces derniers sujets, une mortalité fut dırectement attribuable à cette technique tandıs que dans un autre cas, elle peut avoir contribué à la mort du patient. Dans les deux cas, ll y eut formation d'un hématome à l'endroit de l'injection et dans un 1 l y eut en plus hémorragie médıastinale. Par conséquent les injections sous-clavières, à titre de procédure de routıne ont été abandonées dans notre service d'anesthésie, bien qu'elles soient encore employées dans certains cas d'urgence où le risque associé rend la technique justifiable. 


\section{RúsUMÉ}

Cannulation of the subclavian vein, as first described by French authors, is easily performed in the following steps:

1. A wheal is rassed below the clavicle, at the junction of the inner and middle thirds of the bone.

2. A 3-inch no. 18 needle, attached to a syringe, is inserted through the wheal in a medial, cranial and dorsal durection. Proportionately smaller needles are used in children.

3. With suction applied to the syringe, the needle is slowly advanced, huggin? the under surface of the clavicle, until the costoclavicular ligament is piercec This ligament yields with the feel of the dura mater.

4. A few millimetres beyond this point the vein is entered and the syringe suddenly fills with blood.

5 Aspiration in the four quadrants assures that the entire bevel is within the lumen The syringe is exchanged for the tubing of the intravenous drip and the latter is secured by a prece of tape to prevent drag on the needle. Aspiration is repeated following each change of position and prior to the injection of any drug to verify the correct position of the needle.

On the basis of the first hundred clinical cases, it can be stated that cannulation may be performed with great ease in approximately 95 per cent of the cases.

\section{ADDENDUM}

Since in isolated instances severe complications may result from its use, the technique is not recommended as an elective procedure. In emergency or disaster situations it may, however, prove life saving

\section{BIBLIOGRAPHIE}

1. Aubainac, $R$ Linjection intravemeuse sous-clavière Avantages et technque Presse méd, 601456 (1952)

2 Ké́ri-Szántó, M The Subclavian Vem A Constant and Convenient Intravenous Injection Site Arch Surg, 72179 (1956)

3 Antia, N H Infusions through the Cephalic Vein Brit Med J, 4940652 (1955). 